\title{
Going beyond traditional roughness metrics for floor tiles: Measuring topography down to the nanoscale
}

\author{
Ruikang Ding ${ }^{1}$, Abhijeet Gujrati ${ }^{1}$, Matthew M. Pendolino ${ }^{1}$, Kurt E. Beschorner ${ }^{1,2}$, Tevis D. B. Jacobs ${ }^{1 \#}$ \\ ${ }^{1}$ Department of Mechanical Engineering and Materials Science, University of Pittsburgh, PA, USA \\ ${ }^{2}$ Department of Bioengineering, University of Pittsburgh, PA, USA \\ ${ }^{\#}$ corresponding author \\ Ruikang Ding, RUD12@pitt.edu \\ Abhijeet Gujrati, abg30@pitt.edu \\ Matthew M. Pendolino,_mmp70@pitt.edu \\ Kurt E. Beschorner, beschorn@pitt.edu \\ Tevis D. B. Jacobs, tijacobs@pitt.edu
}

The published version of this article can be found at: doi.org/10.1007/s11249-021-01460-8

Full citation information is as follows:

Ding, R., Gujrati, A., Pendolino, M.M. et al. Going Beyond Traditional Roughness Metrics for Floor Tiles:

Measuring Topography Down to the Nanoscale. Tribol Lett 69, 92 (2021). https://doi.org/10.1007/s11249-

021-01460-8

\begin{abstract}
Slipping is a major cause of injury and hospitalization in the United States and globally. Slipping occurs when the instantaneous friction between the shoe and floor is less than the required friction. While floor roughness is a key factor contributing to friction, prior investigations have primarily used stylus profilometry, which is incapable of measuring roughness at small scales, below approximately 1 micron in lateral size. In the present research, the smallscale roughness was quantified using cross-section scanning electron microscopy (SEM). Three different flooring materials were investigated, including tiles of ceramic and two different types of quarry stones, whose friction coefficients had been previously characterized. The surfaces were cross-sectioned, imaged at magnifications from 250 to 100,000 times, and then the surface profiles were extracted using image analysis. The SEM topography was combined with stylus profilometry measurements, using the power spectral density (PSD), to achieve multi-scale characterization of features ranging from a scan size of $4 \mathrm{~mm}$ down to a resolution of $10 \mathrm{~nm}$. The results demonstrate meaningful differences in topography at different length scales, where surfaces with widely varying roughness at one scale were indistinguishable at another. The measurements further showed that floor-tile roughness has self-affine fractal-like character, with hierarchical roughness extending from the micron-scale down to the nanoscale, much of which is undetectable using conventional techniques. Overall, this research supports the investigation of small-scale roughness as a potential missing factor in the understanding of floor topography and its causal effect on slip-and-fall accidents.
\end{abstract}

Keywords: Slipping · Shoe-floor friction · Coefficient of friction COF · Power spectral density PSD 


\section{Introduction}

\subsection{The Importance of Slips, Trips, and Falls}

Falls due to slipping and tripping are a major cause for occupational injuries at work in the United States as well as other developed countries. For example, a 2018 report from the U.S. Department of Labor showed that falls contributed to about $27 \%$ of all non-fatal occupational injuries leading to days away from work, affecting more than a quarter of a million workers [1]. In other developed countries like the UK and Sweden, slips, trips, and falls contributed to $20-40 \%$ of total occupational injuries [2,3]. In 2007, the related medical cost in the United States was $\$ 7.7$ billion [4, 5]. In 2019, injuries due to falls on the same level were about $\$ 10.4$ billion [6]. Among all fall injuries, slipping is a very common initiating event. Previous investigations show that slipping accounts for over $40 \%$ slip/trip/fall-related injuries [3,7]. In a study on slip events in US restaurants, Verma et al. found that a full-time worker experienced 2.6 major slips per year on average [4]. Overall, slipping is a common occupational accident, and requires continuing research to reduce its incidence. Slipping occurs, when the available coefficient of friction (COF) between the shoe and the floor is less than the required COF for gait (which has a mean between 0.17-0.22 for level and straight walking) [8-10]. While friction does not always vary linearly with load, there is prior work supporting the relevance of COF to slipping incidence $[8,11]$. Furthermore, it is common practice in the shoe-floor community to use $\mathrm{COF}$ as a key metric; therefore, this convention will be used throughout this paper. In a slipping scenario, the interaction involves the surface of the elastic shoe rubber and the surface of the (comparatively rigid) floor tile, often with a lubricant or contaminant layer between them. It should be noted that various factors influence shoe-floor friction, including floor tile roughness [12,13], lubricant conditions [13], testing conditions such as the slipping speed [14, 15], and the shoe-sole pattern design $[13,16]$. The lubrication regime relevant to shoe-floor-contaminant slips, which is described by the Stribeck curve [14, 17], can influence the parameters relevant to friction $[16,18]$. For treaded shoes with sufficient channels, slipping commonly occurs in the boundary lubrication regime [19-21]. Therefore, this investigation focuses specifically on these conditions and the effect of surface topography of flooring tiles on this regime.

\subsection{Conventional Roughness Metrics Predict Behavior in Some Contexts, But are Generally Insufficient to Predict Friction}

Conventional investigations into the contribution of floor roughness on COF typically follow national and international standards for topography measurement, such as ISO-4287 [22] and ASME B46 [23], and therefore focus mainly on scalar parameters to describe the topography. In these studies, a sharp-needle stylus profilometer is used to measure the surface height as a function of lateral position (which is called the "traced profile"). Per ISO standards [24], Gaussian filters are applied to the traced profile to separate it into a linear combination of three contributions from different size scales: (1) the longest-wavelength contribution is deemed the "waviness" $W$; (2) the mediumwavelength contribution to topography is called the "roughness" $R$; and (3) the shortest-wavelength contribution is deemed to be measurement noise and is discarded. The exact cutoff for these regions can vary as described in Ref. [25], but a common choice is as follows: topography with lateral scales larger than $800 \mu \mathrm{m}$ is considered waviness; size scales between 2.5 and $800 \mu \mathrm{m}$ are considered roughness; and any topography with lateral scales less than $2.5 \mu \mathrm{m}$ is discarded as noise. Then statistical parameters are computed from the filtered height data, such as average deviation of the waviness from the midline $W_{a}$ or root-mean-square (RMS) deviation of the roughness from the midline $R_{q}$. Finally, statistical methods are typically used to derive empirical relationships between the scalar topography parameters and the COF. For example, Derler et al. measured the surface topography of flooring and found correlations of barefoot friction with the roughness parameters $P z$ (average height difference between highest peaks and lowest valleys) as well as with the slope of surface peaks [26]. Additionally, for shoe-floor friction, Chang et al. measured

roughness parameters (e.g. $R_{a}, R_{p m}, \Delta_{a}$ ) and waviness parameters (e.g. $W_{t m}, W \Delta_{q}$ ) and assessed their contribution to COF using a multiple linear regression model. This study found that utilizing multiple predictors substantively 
improved the fit of the regression model, although the degree of improvement was dependent on cutoff wavelength and lubricant conditions [27]. In another study about waviness, Chang et al. [28] found that waviness has high correlation coefficients with COF when the contamination is very viscous, while roughness is highly correlated with COF when the contamination has low viscosity. The authors reasoned that the shoe rubber is not likely to penetrate the highly viscous contamination layer such that hydrodynamic forces will become increasingly relevant over surface forces.

While the prior investigations demonstrate important correlations between topography parameters and COF under certain circumstances, the correlation is usually weak with significant scatter in the data, especially when the difference in roughness between two surfaces is small [27-29]. Additionally, some surfaces with clear differences in roughness show no statistical difference in COF depending on testing conditions $[13,15]$. Furthermore, even where clear trends exist for a certain material and set of conditions, these trends are often not generalizable to other materials and conditions [30, 31]. In summary, existing scalar roughness and waviness parameters from stylus profilometry are insufficient to predict friction with high accuracy.

\subsection{Recent Literature Showing the Importance of Small-Scale Roughness}

An expanding research area focuses on the multi-scale nature of surface topography. Investigations of many kinds of natural surfaces including coastlines [32], mountain topography [33], atomic surfaces [34], and fracture surfaces [3537] indicate that surfaces are often geometrically hierarchical and are sometimes fractal-like across many scales. This multi-scale surface topography can be usefully described by the power spectral density (PSD) [38], which separates out the contributions to topography from different lateral length scales. The analytical theory of Persson has demonstrated how the PSD can be used to predict tribological properties of a soft, elastic body in contact with a hard rough surface $[35,39]$. The hysteresis friction can be computed using continuum contact mechanics, applied over all scales. This integration over the PSD necessarily depends strongly on the smallest scales at which roughness is present on the surface, implying that the small-scale of roughness is significant for determining macroscopic friction [39-41]. Even classic single-asperity models of friction by Moore predict the asperity slope to be strongly related to friction [42], and it has been shown that the values of RMS slope and curvature of surfaces with multi-scale roughness are strongly dependent on the scale at which they are measured. Both of these pictures of rough friction imply that, for multi-scale surface roughness, the large-scale friction will be strongly influenced by the short-wavelength (highfrequency) topography components.

However, characterizing small-scale roughness is not easy. Prior measurements of floor-tile topography (e.g. Refs. $[13,15,27,28,43])$ have used stylus and optical profilometry. The former is limited in lateral scale by tip artifacts, the latter by the diffraction of light; neither contains any information at lateral scales smaller than approximately $1 \mu \mathrm{m}$. For smooth materials, atomic force microscopy (AFM) is common for measuring small-scale topography, and this has been applied to walking surfaces through the measurement of small-scale topography on a single asphalt particle [40]. However, the limited height of the AFM tip and the limited scanning range in the vertical direction [44] constrain the use of AFM to surfaces with less than approximately $20 \mu \mathrm{m}$ in height variation. Most floor tiles contain height variations larger than this, and thus limit the use of AFM. Some investigations (e.g. Ref. [45]) have imaged floor tiles using SEM, but these have rendered mostly qualitative results, with no quantitative roughness measurements extracted from that imaging. In summary, the small-scale topography of floor tiles is almost completely unknown.

\subsection{Purpose Statement for This Research}

The purpose of this investigation was to quantitatively characterize roughness of three types of floor tiles across many length scales, including the typically unmeasured sub-micrometer scale. Multi-scale surface roughness was characterized by combining (conventional) stylus profilometry measurements with (unconventional) cross-section SEM analysis. This enabled the investigation into how roughness parameters varied across length scale, especially in the context of recent models showing the importance of small-scale roughness for friction. 


\section{Experimental Methods}

\subsection{Flooring Materials Used in this Research and Brief Overview of Prior Methods from Jones et al.}

This investigation examined the surface topography of floor tiles in the context of prior measurements of shoe-floor friction. The flooring materials used in this research included one ceramic floor tile, and two different types of floor tiles typically called quarry stone (they are designated here as "quarry 1" and "quarry 2"). The shoe material used in the shoe-floor friction studies was synthetic rubber, and was labeled as "slip-resistant" by the manufacturer. The measured COF between various shoes and these floor tiles were previously published in research by Jones et al. [13]. The ceramic tile (ADJF250803, ASTM) was a reference tile that is used in the reference standard ASTM F2508-12a [46]. The quarry 1 (0T01881P, Daltile, Dallas, TX, USA) and quarry 2 (01 010 SM 1, Summitville, OH, USA) samples are commercial tiles, where the full composition is a trade secret, but they are known to include $15-25 \mathrm{wt} \%$ quartz. The quarry 2 sample also included abrasive grits on the surface, presumably to improve slip resistance.

Briefly, the previous study measured the COF using a force plate (Bertec, FP4060, Columbus, OH, USA) and a portable slip simulator [47]. The shoe was slid against the floor tile in the presence of canola oil (65.4 cP) [13] to simulate an occupational slipping incident in the presence of an oily contaminant. The slipping dynamics were captured using a force plate to measure normal and lateral forces. The load was $250 \mathrm{~N}$ on average, and the sliding speed was $0.5 \mathrm{~m} / \mathrm{s}$. All three floor tiles were tested in the same conditions, so that variation of COF between these three floor tiles was mainly due to variation of surface topographic characteristics or roughness. The shoes used in this study were slip-resistant shoes with tread channels and the data from this study, therefore, is presumed to operate in boundary lubrication.

\subsection{Characterization of Roughness Using a Stylus Profilometer}

Traditional surface topography measurements were performed in the investigation by Jones et al [13] using a stylus profilometer. The line profiles were measured with a scan length of $4.0 \mathrm{~mm}$ and a point spacing of $0.5 \mu \mathrm{m}$ over three different locations and orientations at one position for each material using a contact profilometer (Surtronic S-100, TaylorHobson, AMETEK, Leicester, England). For each material, six surface line profile samples were collected. The mean value of average roughness $\boldsymbol{R}_{\boldsymbol{a}}$ and root-mean-square slope (of the "roughness" profile) $\boldsymbol{R}_{\boldsymbol{\Delta} \boldsymbol{q}}$ were computed from these profiles with a $0.8 \mathrm{~mm}$ Gaussian frequency cutoff to remove large-wavelength content.

\subsection{Characterization of Roughness by SEM}

To measure the small-scale roughness, the samples were cross-sectioned and examined in a scanning electron microscope. Sample preparation for SEM characterization mostly followed a typical metallographic sample preparation technique. First, floor tiles (Fig. 1a) were cut to small bars $\left(1.5 \times 1.5 \times 10 \mathrm{~cm}^{3}\right)$ using a high-speed table saw (Model 65, Powermatic Tools, La Vergne, Tennessee, USA) and then to small squares $\left(1.5 \times 1.5 \times 0.5 \mathrm{~cm}^{3}\right)$ using a low-speed diamond saw (TECHCUT 4 ${ }^{\mathrm{TM}}$, Allied High Tech Products Inc, Compton, California, USA) (Fig. 1b). Afterwards, these small pieces were cold-mounted at room temperature using a mounting epoxy (EpoxySet Resin \& Hardener, Allied High Tech Products Inc, Compton, California, USA) in which the ratio of epoxy to hardener was 10 : 1.2 by weight. The sample mounting protected the edges of the floor-tile samples from being rounded or cracked during grinding and polishing. The mounted samples were ground by hand and with a rotating grinder (TWINPREP $5^{\mathrm{TM}}$, Allied High Tech Products Inc, Compton, California, USA) with silicon carbide paper at grits of 120, 320, 600, and 1200 . Then the samples were polished using a rotating wheel (LaboPol-25, Struers, Cleveland, Ohio, USA), with slurries of progressively smaller grit particles: $6-\mu \mathrm{m}$ poly-diamond, $1-\mu \mathrm{m}$ poly-diamond, and $50-\mathrm{nm}$ colloidal alumina. After grinding and polishing, the cross-section surfaces of these sample pieces had a mirror-like finish without visible scratches. To reduce charging in SEM, the non-conductive epoxy mounting was removed from the sample prior to imaging. This was done by making cuts in the epoxy far from the surface of interest, and then gently breaking the 
remaining epoxy away from the hard surface. Imaging before and after epoxy removal also verified that the surface of interest was not visibly damaged or modified in the process.

(a)

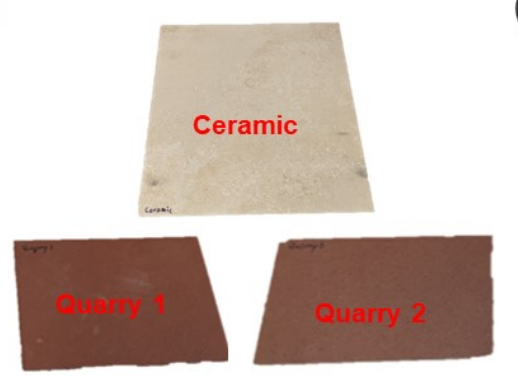

(b)

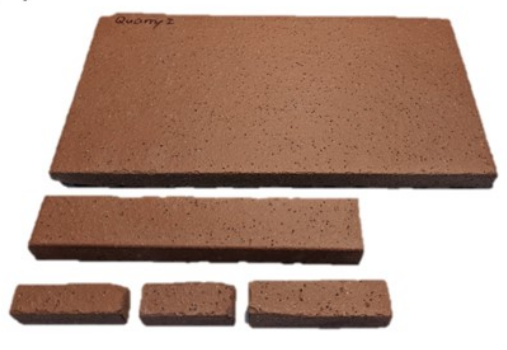

(c)

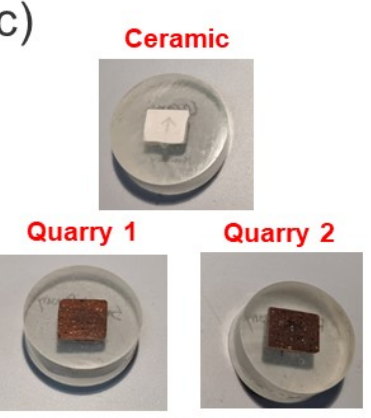

(d)

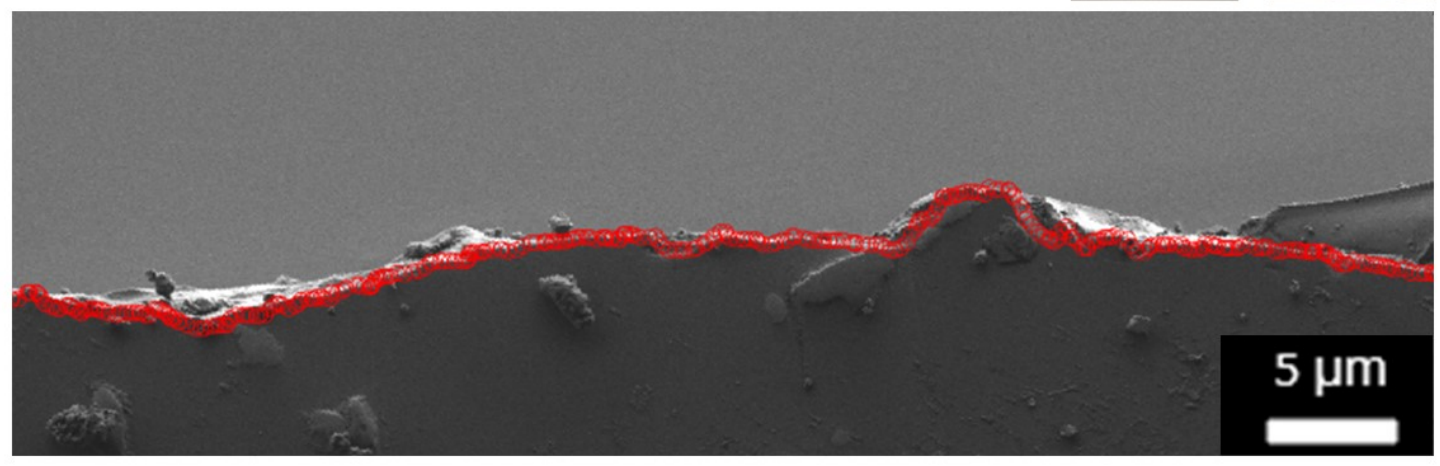

Fig. 1 The small-scale roughness of floor-tile samples was measured with cross-section SEM. Samples of common floor tiles (a) were sectioned (b) and then mounted in epoxy resin (c). After grinding and polishing, the edge representing the original walking surface was imaged in SEM (d) and the line contour of the surface was extracted (red circles).

The cross-sections of the floor surface were observed using a scanning electron microscope (Sigma 500VP, Zeiss, Oberkochen, Germany) with the secondary-electron detector, as shown in Fig. 1d. Since the stone floor-tile materials were non-conductive, a low operating voltage was used $(1 \mathrm{kV})$, with a small working distance $(<4 \mathrm{~mm})$. Also, conductive tape was applied to the sample, up to but not obscuring the surface of interest. Ten images were collected and analyzed for each sample at three different magnifications: $\times 250, \times 5000$, and $\times 100,000$. The mounting, grinding, and polishing process is not expected to affect the topography based on a prior investigation [48] into small-scale topography, which found no statistical difference in topography between cross-section samples and side-view measurements of the same materials that were not sectioned. This lack of modification of surface topography by the sample preparation process is further corroborated by the good agreement between stylus measurements (performed on the un-sectioned samples) and SEM-measured topography from the sectioned sample, as presented later in the Discussion section.

\subsection{Combining Roughness Measurements Using the Power Spectral Density}

The basic methodology for digital image analysis was identical to that for TEM image analysis described in Refs [48, 49], and used custom Matlab scripts. The surface contours in the SEM images were manually traced point by point (red circles in Fig. 1d). Because of the large depth of field in the SEM, various potential line contours were observed, including some surfaces that were clearly out of focus (and therefore out of the roughness plane that was being characterized). Here, only the in-focus surface contour was traced, as indicated by red circles in Fig. 1d. While this practice may lead to some subjectivity in terms of which features are traced, and may include various features with different out-of-plane positions, it has been shown in Ref. [48] that these effects do not have a meaningful effect on the measured statistics of topography. Any obvious contamination was avoided. In limited cases, there were adjacent 
points traced with the same lateral coordinate and different height coordinates; these points were eliminated because the calculations require a well-behaved function (i.e., a single $y$ value for each $x$ value). In total, three materials were measured, each at three magnifications and each of those with ten different locations, for a total of 90 different SEM measurements of small-scale topography.

All extracted profiles were analyzed by computing their PSD, following the procedures defined in Ref. [49]. The raw profiles were tilt-corrected and readjusted to have a mean height of zero by subtracting a linear fit. Also, a Hann window was applied to eliminate artifacts from the endpoints of a non-periodic measurement in a periodic analysis [38]. This is a common step in spectral analysis and is defined by the windowing function: [38, 50]

$$
W_{(x)}=\left(\frac{2}{3}\right)^{\frac{1}{2}}\left(1-\cos \frac{2 \pi x}{L}\right)
$$

where $L$ is the sampling length of the profile. The PSD is computed as the square of the Fourier Transform of the line scan with height $h(x)$ over lateral position $x$ as: [38]

$$
C(q)=L^{-1}|\tilde{h}(q)|^{2}=L^{-1}\left|\int_{0}^{L} h(x) e^{-i q x} d x\right|^{2}
$$

where $\tilde{h}(q)$ is the Fourier transformation of the height, a function of wavevector $q$ that is related to wavelength $\lambda$ by $q=\frac{2 \pi}{\lambda}$. Because the stylus and SEM measurements produced one-dimensional line profiles, the one-dimensional PSD was computed, which is often designated $C^{1 D}(q)$ and which has units of $\left[\mathrm{m}^{3}\right]$. The $C^{1 D}$ is symmetric about $q=0$ and so only the positive-frequency domain is shown. A single PSD is computed from each of the topography measurements. For PSD curves computed from profiles measured by stylus profilometry, artifacts caused by the finite size of the scanning tip are removed. Specifically, the tip radius is known to cause blunting of sharp convex features and also the presence of sharp kinks in concave features [51,52]. Quantitatively, a "reliability cutoff" can be computed, where all data in the PSD above a critical wavevector $q_{c}$ is deemed unreliable. The cutoff $q_{c}$ can be determined by the following equation: [38]

$$
\left[h_{r m s}^{\prime \prime}\left(q_{c}\right)\right]^{2}=\frac{1}{\pi} \int_{0}^{q_{c}} q^{4} C(q) d q=\left(\frac{c}{R}\right)^{2}
$$

where $h_{r m s}^{\prime \prime}\left(q_{c}\right)$ is the RMS curvature computed by integrating PSD curves beginning from $q=0$ to $q=q_{c}, R$ is the radius of curvature of the tip, and $c$ is a constant of order unity. In this study, $R$ is evaluated to be $5 \mu \mathrm{m}$ for a typical stylus probe tip and $c$ is set to 0.5 [49].

Finally, all of the individual PSDs from each topography measurement were combined into a single PSD for the surface. This was accomplished using the arithmetic average of the reliable portions of all the PSDs measured on a single material. The result is a comprehensive PSD curve that describes the statistics of the surface over all measured length scales.

\subsection{Computing Roughness Parameters}

Instead of computing traditional roughness parameters $\left(R_{a}, W_{q}\right.$, etc. $)$ which describe a specific range of length scales, we choose to report the RMS height $h_{r m s}$, the RMS slope $h_{r m s}^{\prime}$, and the RMS curvature $h_{r m s}^{\prime \prime}$. These parameters can be computed from individual measurements, and thus can be used to compare how these values change with size scale; or they can be computed from the whole multi-scale topography, in which case they represent the most accurate possible measurement of the "true" values of height, slope, and curvature of the surface. This analysis is discussed in Refs. $[38,49]$ and is only briefly repeated here.

For analysis of real-space profiles, the RMS parameters are computed as follows [49]:

$$
\begin{gathered}
h_{r m s}{ }^{2}=\frac{1}{L} \int_{0}^{L} h(x)^{2} d x \\
h_{r m s}^{\prime}{ }^{2}=\frac{1}{L} \int_{0}^{L}\left(\frac{d h}{d x}\right)^{2} d x \\
h_{r m s}^{\prime \prime}{ }^{2}=\frac{1}{L} \int_{0}^{L}\left(\frac{d^{2} h}{d x^{2}}\right)^{2} d x
\end{gathered}
$$

For discrete topography data, we used the first-order trapezoidal approximation to compute these parameters. The equations are: [49] 


$$
\begin{aligned}
& h_{r m s}{ }^{2}=\frac{1}{L} \sum_{i=2}^{N}\left[\frac{1}{2}\left(h_{i}^{2}+h_{i-1}^{2}\right)\left(x_{i}-x_{i-1}\right)\right] \\
& h_{r m s}^{\prime}{ }^{2}=\frac{1}{L\left(\frac{N-1}{N}\right)} \sum_{i=3}^{N}\left[\frac{1}{2}\left(\left(\frac{h_{i}-h_{i-1}}{x_{i}-x_{i-1}}\right)^{2}+\left(\frac{h_{i-1}-h_{i-2}}{x_{i-1}-x_{i-2}}\right)^{2}\right)\left(x_{i}-x_{i-1}\right)\right] \\
& h_{r m s}^{\prime \prime}{ }^{2}=\frac{1}{L\left(\frac{N-2}{N}\right)} \sum_{i=4}^{N}\left[\frac{1}{2}\left(\left(\frac{\frac{h_{i}-h_{i-1}}{x_{i}-x_{i-1}}-\frac{h_{i-1}-h_{i-2}}{x_{i-1}-x_{i-2}}}{\left(x_{i}-x_{i-2}\right) / 2}\right)^{2}+\left(\frac{\frac{h_{i-1}-h_{i-2}}{x_{i-1}-x_{i-2}}-\frac{h_{i-2}-h_{i-3}}{x_{i-2}-x_{i-3}}}{\left(x_{i-1}-x_{i-3}\right) / 2}\right)^{2}\right)\left(x_{i-1}-x_{i-2}\right)\right]
\end{aligned}
$$

where $N$ is the number of data points.

While Eqs. 4 and 5 apply to individual measurements of surfaces, they cannot be used to compute parameters from the whole-scale roughness. Instead, this must be done using the full multi-scale PSD, with Parseval's theorem dictating the equivalence of moments computed in real space or frequency space. Thus, the full multi-scale averaged PSD can be integrated to compute values for $h_{r m s}, h_{r m s}^{\prime}$, and $h_{r m s}^{\prime \prime}$. The integration over the positive-frequency domain takes the following form $[38,49]$ :

$$
\begin{gathered}
h_{r m s}{ }^{2}=\frac{1}{\pi} \int_{0}^{\infty} C(q) d q \\
h_{r m s}^{\prime 2}=\frac{1}{\pi} \int_{0}^{\infty} q^{2} C(q) d q \\
h_{r m s}^{\prime \prime 2}=\frac{1}{\pi} \int_{0}^{\infty} q^{4} C(q) d q
\end{gathered}
$$

\section{Results and Discussion}

\subsection{COF \& results from stylus profilometry}

The coefficient of friction is reported for shoe-floor slipping measurements on these three floor-tile materials in Table 1. Also listed are the conventional roughness parameters average roughness $R_{a}$ and root-mean-square slope $R_{\Delta q}$. While both of these roughness parameters are expected [27, 29] to exhibit positive correlation with COF, these correlations are not borne out here. Despite large variations in average roughness (the smallest value is $41 \%$ smaller than the largest value), the variation of COF is slight (the smallest is just $10 \%$ less than the largest). Moreover, the "smoothest" material (lowest values of $R_{a}$ and $R_{\Delta q}$ ) exhibits the highest friction, while the "roughest" material (highest values of $R_{a}$ and $R_{\Delta q}$ ) exhibits an intermediate value of friction. These results run counter to those expected correlations and indicate that conventional scalar roughness parameters may not be sufficient to predict shoe-floor friction.

Table 1 Average values are reported for shoe-floor COF on the three types of floor tiles as measured with an oily contaminant

\begin{tabular}{cccc}
\hline Floor Tiles & Ceramic & Quarry 1 & Quarry 2 \\
\hline COF & $0.450 \pm 0.126$ & $0.355 \pm 0.076$ & $0.403 \pm 0.089$ \\
$R_{a}$ & $3.82 \pm 0.19 \mu \mathrm{m}$ & $4.74 \pm 0.72 \mu \mathrm{m}$ & $6.51 \pm 1.83 \mu \mathrm{m}$ \\
$R_{\Delta q}$ & $0.584 \pm 0.023$ & $0.644 \pm 0.011$ & $0.672 \pm 0.046$ \\
\hline
\end{tabular}

Also reported are the mean values of commonly measured scalar roughness parameters $R_{a}$ and $R_{\Delta q}$ from stylus profilometry (with a 0.8-mm cutoff wavelength). Table adapted from Ref. [13]

\subsection{SEM Observations}

Cross-section SEM images demonstrate the surface topography at various size scales, as shown in Fig. 2. Some contamination is visible including, e.g., a fiber in Fig. 2a1, but is readily identified and not included in the topography 
measurement. The imaging also contains some charging effects due to the non-conductive nature of the stone floortile materials. This effect is most prominent at the highest magnification and contributes some rounding of sharp-point features. The effect of charge precluded imaging at magnifications higher than $\times 100,000$, and may introduce artifacts at the very smallest scales. Even so, the resolution is high enough to measure topography for these samples on scales that are significantly smaller than conventional topography techniques.

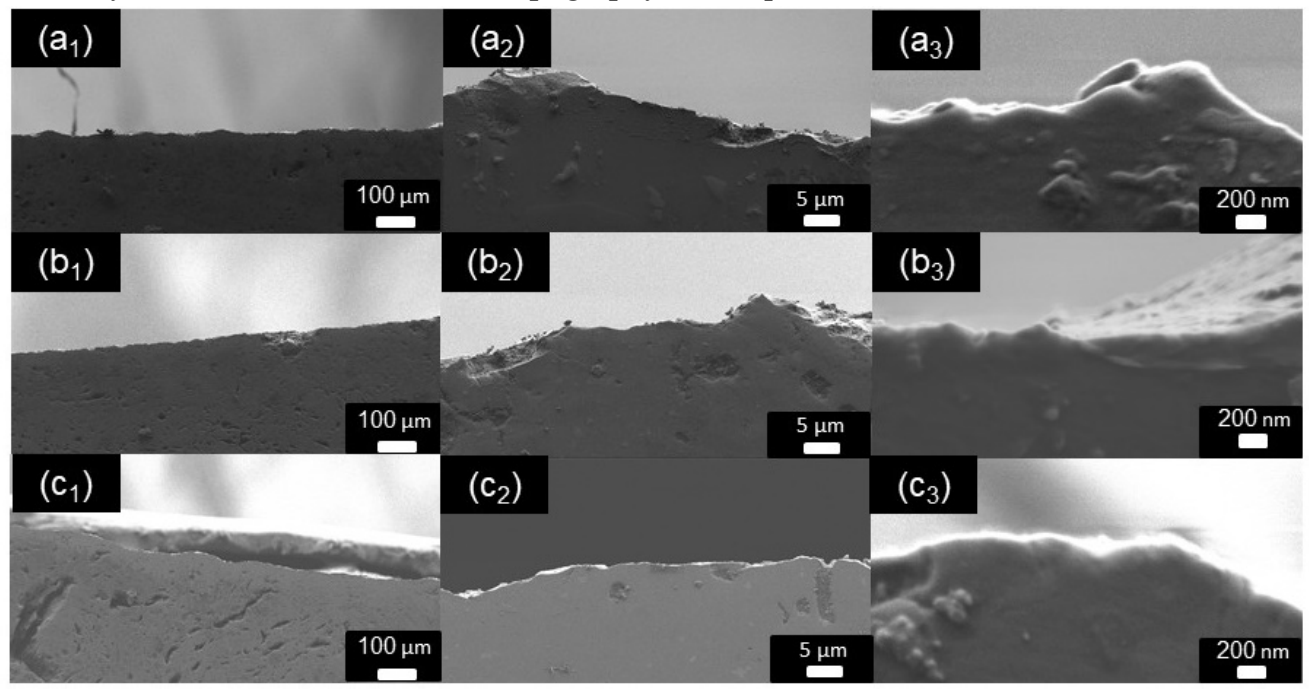

Fig. 2 Representative cross-section images for the surfaces are shown for samples of ceramic (a), quarry 1 (b), and quarry 2 (c) flooring. Images are shown at instrument magnifications of $\times 250$ (left), $\times 5000$ (center), and $\times 100,000$ (right)

\subsection{Computing and Comparing Roughness Parameters from Real-Space Measurements}

The RMS height, slope, and curvature have been computed from each topography measurement, including all SEM and stylus data. The results are displayed in Fig. 3, and demonstrate the dependence of these parameters on the length scale over which they are measured. The RMS height is presented on a $\log$ scale as a function of sampling length $L$, since it is most strongly affected by large-scale features. The RMS slope and RMS curvature are presented as a function of measurement resolution $l$, defined as the mean spacing between measured points in stylus or SEM profile, to reflect their dependence on small-scale features. The results can be analyzed using the variable bandwidth method (VBM) [53, 54], which relates these parameters as $h_{r m s} \propto L^{H}$, where $H$ is the Hurst exponent [53]. Furthermore, it can be shown mathematically $[35,38]$ that the RMS slope and RMS curvature should vary with pixel size as $h_{r m s}^{\prime} \propto l^{(H-1)}$ and $h_{r m s}^{\prime \prime} \propto l^{(H-2)}$, respectively. Therefore, all of these parameters have been fit using a power-law relationship to extract $H$. Using RMS height, the best-fit values for Hurst exponent $H$ are: $0.73 \pm 0.08$ for the ceramic, $0.83 \pm 0.07$ for quarry 1 , and $0.86 \pm 0.06$ for quarry 2 . Using RMS slope, the best-fit values for $H$ are: $0.98 \pm 0.05$ for ceramic, $0.96 \pm 0.05$ for quarry 1 , and $0.95 \pm 0.05$ for quarry 2. Using the RMS curvature, the best-fit values for $H$ are: $0.98 \pm$ 0.11 for ceramic, $0.94 \pm 0.10$ for quarry 1 , and $0.93 \pm 0.10$ for quarry 2 . Note that since the values of $h_{r m s}^{\prime}$ are approximately constant (scaling exponent near zero), the measured dependence on $H$ is not statistically significant and has a very low $R^{2}$ value; however, the fits from $h_{r m s}$ and $h_{r m s}^{\prime \prime}$ are much more strongly correlated to $H$ (higher $R^{2}$ ). If we take all of these scaling analyses together, and average the various extracted values for each material, we get the following results for $H=0.90$ for ceramic, $H=0.91$ for quarry 1 , and $H=0.90$ for quarry 2. Overall, the scaling behavior extracted from real-space measurements appears similar for all three materials, despite differences in the absolute values of individual roughness metrics. Assessment of the scaling behavior of topography can be performed even more accurately using a spectral analysis of results, as is performed in the following sub-section. 

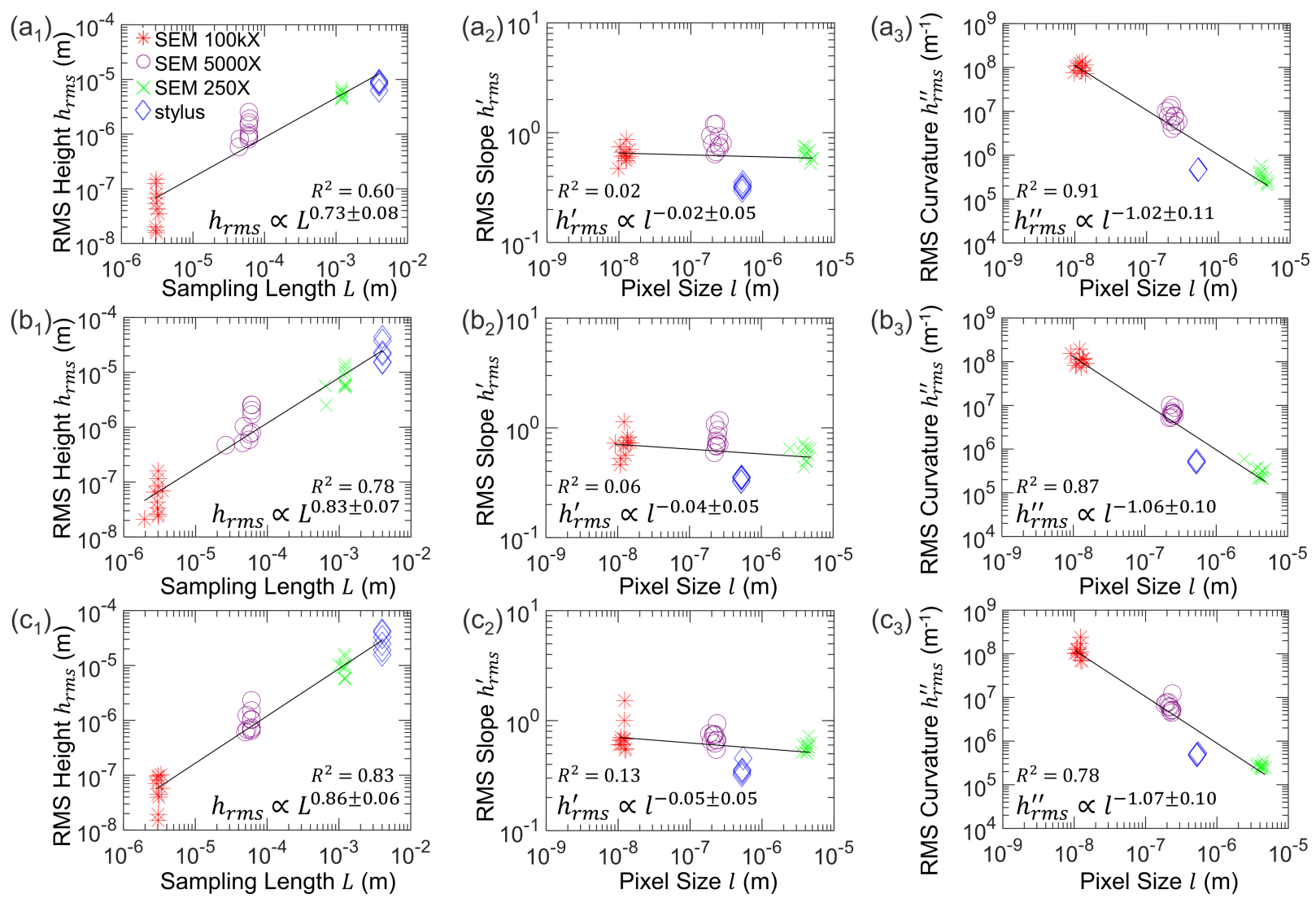

Fig. 3 Roughness parameters calculated from real-space measurements. RMS parameters $h_{r m s}, h_{r m s}^{\prime}$, and $h_{r m s}^{\prime \prime}$ are directly calculated from the surface profiles at different scales which are represented by sampling length $L$ or pixel size $l$ for ceramic (a), quarry 1 (b), and quarry 2 (c). Power-law fitting is applied separately to $h_{r m s}$, $h_{r m s}^{\prime}$ and $h_{r m s}^{\prime \prime}$. The error is defined as $95 \%$ confidence interval of standard error. The coefficient of determination is included in each plot

\subsection{Computing the Power Spectral Density, and Computing Topography Parameters in Frequency Space}

The power spectral density was computed from each individual topography measurement-from stylus and crosssection SEM - and plotted on the same curve (Fig. 4a-c). Finally, all of the individual PSDs were averaged to create one curve for each material, describing the statistics of the whole surface over all measured scales (Fig. 4d). The spectral analysis of the multi-scale surface topography demonstrates that these floor-tile samples exhibit self-affine fractal-like scaling over a large range of size scales. This scaling is described by fitting the self-affine region with $C(q)=C_{0} q^{-\beta}$ and extracting the best-fit exponent. The measured $\beta$ values of ceramic, quarry 1 and quarry 2 are 2.83 $\pm 0.06,2.82 \pm 0.07$, and $2.75 \pm 0.05$, respectively. In the self-affine region, the fractal dimension is $D=(2 n+3-$ $\beta) / 2$ [55], where $n$ is the spatial dimension, and is $n=1$ for a line-scan surface, yielding $D=(5-\beta) / 2$. This fractal dimension is also commonly represented as a function of the Hurst exponent as $D+H=n+1=2$ [56]; therefore, $H=(\beta-1) / 2$. Based on calculated values of $\beta$ of the three materials, $H=0.92 \pm 0.03$ for ceramic, and $H=$ $0.91 \pm 0.04$ for quarry 1 , and $H=0.88 \pm 0.03$ for quarry 2 . These values agree, within experimental uncertainty, with the values of $H$ extracted from the real-space power-law fitting of Fig. 3 ( $H=0.90,0.91$, and 0.90 , respectively). 
(a)

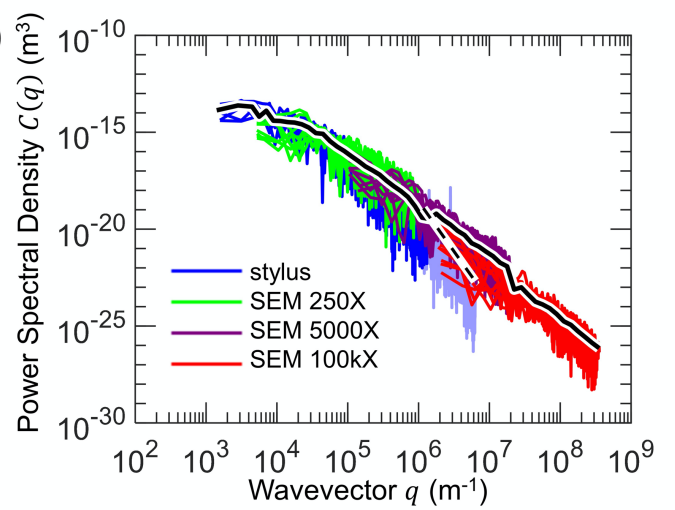

(c)

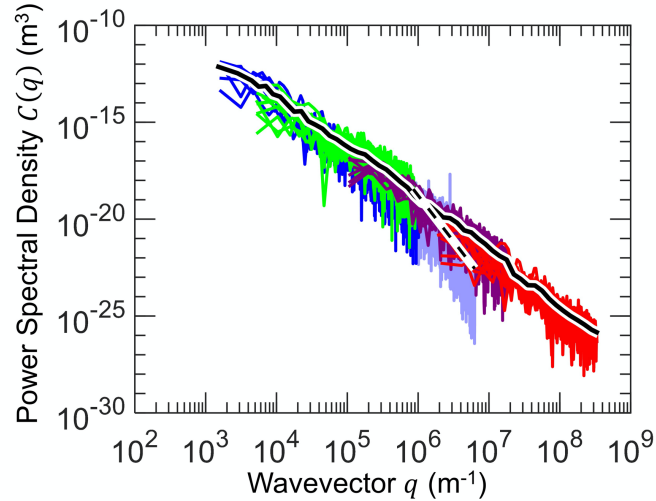

(b)

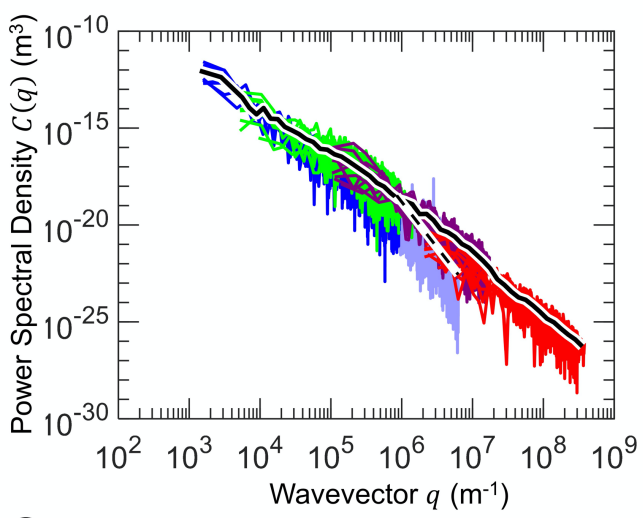

(d)

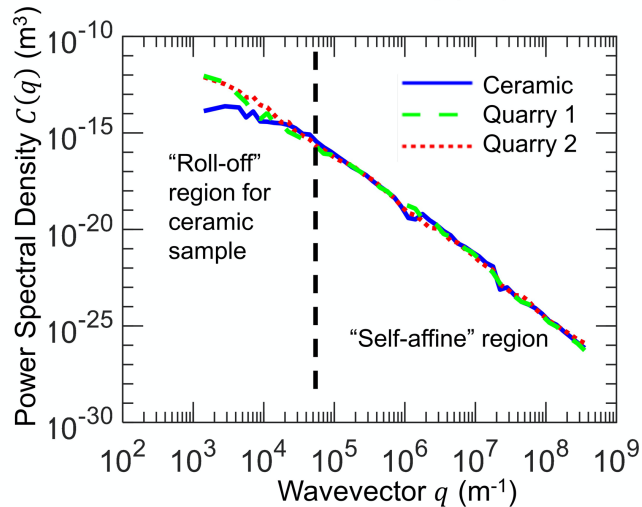

Fig. 4 The topography of all size scales is characterized by the averaged power spectral density of all measurements. Individual PSD curves, plotted as a function of wavevector $q=\frac{2 \pi}{\lambda}$, are shown for ceramic (a), quarry 1 (b), and quarry 2 (c). The colored lines indicate individual measurements, with color corresponding to the technique and magnification (legend), while the black curves indicate the arithmetic average of the individual measurements. The unreliable (artifacted) portion of the stylus measurements (light blue for individual measurements, dashed black line for averaged PSD) deviates significantly from the true topography, as described in the main text. The averaged PSD data aew combined on a single curve (d) to demonstrate the similarity of topography at all but the largest scales

The quarry samples are self-affine over the entire range of measured scales: more than five decades of size scale from a minimum resolution of $10 \mathrm{~nm}$ to a maximum sample size of $4 \mathrm{~mm}$. The ceramic sample is self-affine over almost four decades of size scales, but exhibits "roll-off" behavior at the largest scales, where the slope of the powerlaw scaling changes at approximately $q=5 \times 10^{4} \mathrm{~m}^{-1}(\lambda=100 \mu \mathrm{m})$ from -2.83 in the "self-affine" region to -0.99 in the "roll-off region". The presence of "roll-off" behavior has been well-documented in literature e.g. Refs [35, 57]; however, its origin is not well-understood. Prior observations [35] demonstrate that fractured surfaces often lack rolloff behavior, while agglomerations of particulates tend to demonstrate a clear roll-off point. Furthermore, surface treatments like sand blasting and certain polishing techniques can cause roll-off regions to develop and grow. The precise manufacturing history of these commercial tiles is unknown, and therefore the roll-off cannot be linked to a specific physical origin.

One key insight that emerges from this analysis is that, despite a large difference (41\%) in traditional roughness parameters $\left(R_{a}\right.$ and $R_{\Delta q}$, Table 1), these three flooring materials are indistinguishable when examined at the small scales (right side of Fig. 4d). The scaling behavior of these materials are identical, and it is only the presence of the aforementioned roll-off region that differs between them. This means that, at least in some cases, the large-scale topography is unrepresentative of the small-scale topography. Because conventional (stylus-profilometry) 
measurements of topography are primarily focused on this larger-scale region, they can overemphasize differences and result in very different roughness parameters, even for surfaces that are similar across the majority of length scales. Moreover, some investigations rely on assumptions of self-affinity to extrapolate from large-scale measurements to describe the roughness at the smaller scale. While this would work acceptably for the quarry samples, it would produce erroneous results for the ceramic sample.

A second key insight is that, apart from the roll-off region, all surfaces show self-affine scaling behavior down to the very smallest scales that were measured. This indicates that flooring surfaces can have a significant contribution to roughness from scales that are entirely inaccessible using stylus profilometry. This finding of the similar nature of these three surfaces, despite significant variations in traditional roughness parameters, is particularly important in light of the recent friction literature (discussed in the Introduction) that demonstrates the importance of smaller-scale topography for true contact area, adhesion, and friction. It is not yet clear which scales matter most for shoe-floor friction; therefore, these findings support the need for more comprehensive topography characterization to capture and assess these previously unmeasured small length scales.

\subsection{Computing RMS parameters in frequency space}

Because the traditional roughness metrics (Table 1) fail to accurately capture the variations and similarities between these three materials, we compute RMS parameters for the entire surface at all measured scales using Parseval's law (see Methods). To be clear, this approach does not make any assumptions about fractal or power-law fits to the data; instead, it integrates under the exact PSD curves shown in Fig. $4 \mathrm{~d}$ to compute their various moments. Table 2 shows these measured RMS parameters for the three surfaces. Unlike any real-space calculation, this approach has the advantage of computing RMS parameters using all of the multi-scale data that are available. The root-mean-square height is largest for the quarry 2 , which contains the large-scale abrasive grit, and is smallest for the ceramic surface. This reflects the significant differences in the large-scale topography, but these large-scale differences may not be especially relevant for friction (see Introduction). Note that these values for $h_{r m s}$ are significantly larger than the values of $R_{a}$ (Table 1); however, this simply reflects the fact that $R_{a}$ has had the large-scale features (waviness) filtered out (per ISO 4287 [22]) while $h_{r m s}$ includes all size scales. The parameter $h_{r m s}$ would be analogous to the parameter $P_{q}$ from ISO 4287, if it were possible to measure the surface profile $P$ with large scan size ( $\left.>4 \mathrm{~mm}\right)$ and extremely fine resolution $(<10 \mathrm{~nm})$. We choose to report $h_{r m s}$ here because modeling and simulation literature on shoe-rubber friction indicate that all size scales may be important input for shoe-floor friction $[12,58]$.

Table 2 RMS parameters $h_{r m s}, h_{r m s}^{\prime}$, and $h_{r m s}^{\prime \prime}$ calculated in frequency space from the averaged multi-scale PSD curves for each material

\begin{tabular}{cccc}
\hline Sample & RMS height $h_{r m s}(\mathrm{~m})$ & RMS slope $h_{r m s}^{\prime}$ & RMS curvature $h_{r m s}^{\prime \prime}\left(m^{-1}\right)$ \\
\hline Ceramic & $8.24 \times 10^{-6} \pm 0.74 \times 10^{-6}$ & $0.854 \pm 0.069$ & $7.80 \times 10^{7} \pm 1.15 \times 10^{7}$ \\
Quarry 1 & $2.70 \times 10^{-5} \pm 0.69 \times 10^{-5}$ & $0.816 \pm 0.060$ & $7.11 \times 10^{7} \pm 1.05 \times 10^{7}$ \\
Quarry 2 & $2.71 \times 10^{-5} \pm 0.43 \times 10^{-5}$ & $0.814 \pm 0.059$ & $9.13 \times 10^{7} \pm 1.86 \times 10^{7}$ \\
\hline
\end{tabular}

Uncertainty is computed using standard error propagation

As for the RMS slope and curvature, which have been linked to the adhesion and friction performance of surfaces, these three different floor tiles show no statistical difference in their measured values. The RMS slope of these surfaces, when measured over a large range of size scales, is approximately 0.828 , which corresponds to an angle of 40 degrees. The RMS curvature of these surfaces, measured over the same scales is $8.01 \times 10^{7} \mathrm{~m}^{-1}$ on average, which corresponds to a radius of curvature of about $12 \mathrm{~nm}$. In particular, the RMS slope ( $h_{r m s}^{\prime}$ from Table 2$)$ can be compared to the conventional slope value $\left(R_{\Delta q}\right.$ from Table 1$)$. Using conventional techniques and metrics, the slope is computed to be smaller (mean of the three surfaces of 0.63 ) and more different between surfaces ( $15 \%$ change of largest over smallest); 
while when all scales of topography are measured, the true slope is measured to be larger (mean of 0.83 ) with greater similarity. In the case of the ceramic sample, the true RMS slope was found to be $46 \%$ larger than the slope measured using conventional approaches.

These findings provide one possible explanation for the apparent contradiction raised by the investigation of Jones et al. where differences in roughness did not follow expected correlations with differences in friction. Conventional topography metrics emphasize the large-scale topography, and therefore indicate meaningful differences between these three materials. However, as discussed in the Introduction, the smaller-scale topography is expected to contribute most significantly to friction for the case of soft surfaces like rubber and hard rough surfaces like floor tiles in the boundary-lubrication regime. The lack of a statistically significant difference in the high-q (small length scale) region of the PSD, and the related lack of a difference between the computed parameters of $h_{r m s}^{\prime}$, and $h_{r m s}^{\prime \prime}$, are consistent with the similarity (within 10\%) of values of COF between the three materials. These results are not claimed to prove causation, but rather to provide a possible explanation, and to motivate further investigation into the small-scale topography of floor tiles as a possible route to establishing predictive understanding of shoe-floor friction.

\section{Conclusions}

This study characterized the multi-scale surface roughness of common quarry and ceramic floor tiles using crosssection scanning electron microscopy and stylus profilometry. Results demonstrate that the surface topography of flooring materials is hierarchical and multi-scale, with a significant contribution from scales that are invisible using traditional measurement techniques. Scalar roughness parameters were computed from both real-space and frequencyspace measurements and reflected significant deviations from conventional scalar parameters measured using stylus profilometry. The findings provide one possible resolution to an apparent paradox from prior work where significant differences in topography did not result in meaningful differences in friction; a potential explanation is that the smallscale topography of these three surfaces is nearly identical. These findings support theoretical and simulation literature in suggesting that there may be a significant effect on macroscopic friction due to small-scale topography, and therefore motivate further investigation into topography that is unmeasurable using current techniques.

\section{Acknowledgements}

The authors acknowledge the use of the Nanoscale Fabrication and Characterization Facility (NFCF) in the Gertrude E. \& John M. Petersen Institute of NanoScience and Engineering and Materials Micro-characterization Lab (MMCL) in Swanson School of Engineering. Funding was provided by the National Science Foundation under award number CMMI-1727378.

\section{Authors' contributions}

$\mathrm{RD}$ performed sample preparation, characterization, analysis and writing of the manuscript. AG assisted with characterization and analysis. MMP performed sample preparation. KEB and TDBJ guided the research and revised the manuscript.

\section{Funding}

Funding was provided by the National Science Foundation under award number CMMI-1727378.

\section{Data Availability}

All materials are stored in the lab of Tevis D. B. Jacobs.

The topography data that support the fndings of this study are openly available at the following URLs:

Ceramic: https://contact.engineering/go/6xqx7/

Quarry 1: https://contact.engineering/go/p3zuu/

Quarry 2: https://contact.engineering/go/rptba/ 


\section{Code availability}

The custom codes used in the topography analysis are available upon request.

\section{Declarations}

\section{Conflicts of interest/Competing interests}

The authors declare no conflicts of interest.

\section{References}

1. U.S. department of labor-Bureau of Labor Statistics (BLS).: Employer-Reported Workplace Injuries and Illnesses, 2018. (2019)

2. Leclercq, S., Thouy, S., Rossignol, E.: Progress in understanding processes underlying occupational accidents on the level based on case studies. Ergonomics. 50, 59-79 (2007). https://doi.org/10.1080/00140130600980862

3. Courtney, T.K., Sorock, G.S., Manning, D.P., Collins, J.W., Holbein-jenny, M.A.: Occupational slip, trip, and fall related injuries: can the contribution of slipperiness be isolated? Ergonomics. 44, 1118-1137 (2001). https://doi.org/10.1080/0014013011008553

4. Verma, S.K., Chang, W.R., Courtney, T.K., Lombardi, D.A., Huang, Y., Brennan, M.J., Mittleman, M.A., Ware, J.H., Perry, M.J.: A prospective study of floor surface, shoes, floor cleaning and slipping in US limited-service restaurant workers. Occup. Environ. Med. 68, 279-285 (2011). https://doi.org/10.1136/oem.2010.056218

5. Liberty Mutual Research Institute for Safety.: The Most Disabling Workplace Injuries Cost Industry an Estimated \$52 Billion. https://www.libertymutualgroup.com/about-lm/\%0Acorporate-information/overview (2009). Accessed 2020

6. Liberty Mutual Insurance.: 2019 Workplace Safety Index: The top 10 causes of disabling injuries at work. https://viewpoint.libertymutualgroup.com/article/top-10-causes-disabling-injuries-at-work-2019/ (2019). Accessed 2020

7. Verma, S.K., Lombardi, D.A., Chang, W., Theodore, K., Brennan, M.J.: A matched case-control study of circumstances of occupational same-level falls and risk of wrist, ankle and hip fracture in women over 45 years of age. Ergonomics. 51, 19060-21972 (2008). https://doi.org/10.1080/00140130802558987

8. Hanson, J.P., Redfern, M.S., Mazumdar, M.: Predicting slips and falls considering required and available friction. Ergonomics. 42, 1619-1633 (1999). https://doi.org/10.1080/001401399184712

9. Redfern, M.S., Cham, R., Gielo-perczak, K., Grönqvist, R., Hirvonen, M., Lanshammar, H., Marpet, M., Pai, C.Y.-C.I., Powers, C.: Biomechanics of slips. Ergonomics. 44, 1138-1166 (2001). https://doi.org/10.1080/0014013011008554

10. Strobel, C.M., Menezes, P.L., Lovell, M.R., Beschorner, K.E.: Analysis of the contribution of adhesion and hysteresis to shoe-floor lubricated friction in the boundary lubrication regime. Tribol. Lett. 47, 341-347 (2012). https://doi.org/10.1007/s11249-012-9989-5

11. Iraqi, A., Cham, R., Redfern, M.S., Beschorner, K.E.: Coefficient of friction testing parameters influence the prediction of human slips. Appl. Ergon. 70, 118-126 (2018). https://doi.org/10.1016/j.apergo.2018.02.017

12. Moghaddam, S.R.M., Redfern, M.S., Beschorner, K.E.: A Microscopic Finite Element Model of Shoe-Floor Hysteresis and Adhesion Friction. Tribol. Lett. 59, 1-10 (2015). https://doi.org/10.1007/s11249-015-0570-x

13. Jones, T., Iraqi, A., Beschorner, K.: Performance testing of work shoes labeled as slip resistant. Appl. Ergon. 68, 304-312 (2018). https://doi.org/10.1016/j.apergo.2017.12.008

14. Beschorner, K., Lovell, M., Higgs III, C.F., Redfern, M.S.: Modeling mixed-lubrication of a shoe-floor interface applied to a pin-on-disk apparatus. Tribol. Trans. 52, 560-568 (2009).

https://doi.org/10.1080/10402000902825705

15. Cowap, M.J.H., Moghaddam, S.R.M., Menezes, P.L., Beschorner, K.E.: Contributions of adhesion and hysteresis to coefficient of friction between shoe and floor surfaces: effects of floor roughness and sliding speed. Tribol. - Mater. Surfaces Interfaces. 9, 77-84 (2015). https://doi.org/10.1179/1751584X15Y.0000000005

16. Iraqi, A., Vidic, N.S., Redfern, M.S., Beschorner, K.E.: Prediction of coefficient of friction based on 
footwear outsole features. Appl. Ergon. 82, 102963 (2020). https://doi.org/10.1016/j.apergo.2019.102963

17. Beschorner, K.E., Redfern, M.S., Porter, W.L., Debski, R.E.: Effects of slip testing parameters on measured coefficient of friction. Appl. Ergon. 38, 773-780 (2007). https://doi.org/10.1016/j.apergo.2006.10.005

18. Beschorner, K.E., Hemler, S.L., Moghaddam, S.R.M., Iraqi, A., Redfern, M.S.: Footwear for the prevention of human slips: from friction mechanics to ergonomic solutions. In: "Slips, Trips \& Falls" Conference Madrid 2020 (2020)

19. Singh, G., Beschorner, K.E.: A Method for Measuring Fluid Pressures in the Shoe-Floor-Fluid Interface: Application to Shoe Tread Evaluation. IIE Trans Occup. 2, 53-59 (2014). https://doi.org/10.1080/21577323.2014.919367

20. Hemler, S.L., Charbonneau, D.N., Iraqi, A., Redfern, M.S., Haight, J.M., Moyer, B.E., Beschorner, K.E.: Changes in under-shoe traction and fluid drainage for progressively worn shoe tread. Appl. Ergon. 80, 3542 (2019). https://doi.org/10.1016/j.apergo.2019.04.014

21. Beschorner, K.E., Albert, D.L., Chambers, A.J., Redfern, M.S.: Fluid pressures at the shoe-floorcontaminant interface during slips: Effects of tread \& implications on slip severity. J. Biomech. 47, 458-463 (2014). https://doi.org/10.1016/j.jbiomech.2013.10.046

22. ISO 4287: Geometrical Product Specifications (GPS) - Surface texture: Profile method - Terms, definitions and surface texture parameters. (1997)

23. ASME B46: Committee on Classification and Designation of Surface Qualities. (2005)

24. ISO 16610: Geometrical product specifications (GPS) - Filtration. (2011)

25. Geometrical Product Specifications (GPS) - Surface texture: Profile method - Nominal characteristics of contact (stylus) instruments. (1996)

26. Derler, S., Huber, R., Feuz, H.P., Hadad, M.: Influence of surface microstructure on the sliding friction of plantar skin against hard substrates. Wear. 267, 1281-1288 (2009).

https://doi.org/10.1016/j.wear.2008.12.053

27. Chang, W., Matz, S., Grönqvist, R.: Linear regression models of floor surface parameters on friction between Neolite and quarry tiles. Appl. Ergon. 41, 27-33 (2010).

https://doi.org/10.1016/j.apergo.2009.03.006

28. Chang, W., Grönqvist, R., Hirvonen, M.: The effect of surface waviness on friction between Neolite and quarry tiles. Ergonomics. 47, 890-906 (2004). https://doi.org/10.1080/00140130410001670390

29. Chang, W.R.: The effect of surface roughness and contaminant on the dynamic friction of porcelain tile. Appl. Ergon. 32, 173-184 (2001). https://doi.org/10.1016/S0003-6870(00)00054-5

30. Kim, I., Hsiao, H., Simeonov, P.: Functional levels of floor surface roughness for the prevention of slips and falls: Clean-and-dry and soapsuds-covered wet surfaces. Appl. Ergon. 44, 58-64 (2013).

https://doi.org/10.1016/j.apergo.2012.04.010

31. El-Sherbiny, Y.M., Hasouna, A.T., Ali, W.Y.: Friction coefficient of rubber sliding against flooring materials. ARPN J. Eng. Appl. Sci. 7, 121-126 (2012)

32. Mandelbrot, B.: How Long Is the Coast of Britain? Statistical Self-Similarity and Fractional Dimension. Sci. 156, 636-638 (1967)

33. Candela, T., Renard, F., Klinger, Y., Mair, K., Schmittbuhl, J., Brodsky, E.E.: Roughness of fault surfaces over nine decades of length scales. J. Geophys. Res. Solid Earth. 117, 1-30 (2012). https://doi.org/10.1029/2011JB009041

34. Persson, B.N.J.: On the fractal dimension of rough surfaces. Tribol. Lett. 54, 99-106 (2014). https://doi.org/10.1007/s11249-014-0313-4

35. Persson, B.N.J., Albohr, O., Tartaglino, U., Volokitin, A.I., Tosatti, E.: On the nature of surface roughness with application to contact mechanics, sealing, rubber friction and adhesion. J. Phys. Condens. matter. 17, R1-R62 (2005). https://doi.org/10.1088/0953-8984/17/1/R01

36. Kotowski, P.: Fractal dimension of metallic fracture surface. Int. J. Fract. 141, 269-286 (2006). https://doi.org/10.1007/s10704-006-8264-x

37. Bonamy, D., Ponson, L., Prades, S., Bouchaud, E., Guillot, C.: Scaling exponents for fracture surfaces in homogeneous glass and glassy ceramics. Phys. Rev. Lett. 97, 1-4 (2006).

https://doi.org/10.1103/PhysRevLett.97.135504

38. Jacobs, T.D.B., Junge, T., Pastewka, L.: Quantitative characterization of surface topography using spectral analysis. Surf. Topogr. Metrol. Prop. 5, 013001 (2017). https://doi.org/10.1088/2051-672X/aa51f8

39. Persson, B.N.J.: Theory of rubber friction and contact mechanics. J. Chem. Phys. 115, 3840-3861 (2001). https://doi.org/10.1063/1.1388626

40. Lorenz, B., Oh, Y.R., Nam, S.K., Jeon, S.H., Persson, B.N.J.: Rubber friction on road surfaces: Experiment 
and theory for low sliding speeds. 142, 194701 (2015). https://doi.org/10.1063/1.4919221

41. Yang, C., Persson, B.N.J.: Contact mechanics: Contact area and interfacial separation from small contact to full contact. J. Phys. Condens. Matter. 20, 215214 (2008). https://doi.org/10.1088/0953-8984/20/21/215214

42. Moore, D.F.: The Friction and Lubrication of Elastomers. Pergamon Press, Oxford (1972)

43. Chang, W.R., Kim, I.J., Manning, D.P., Bunterngchit, Y.: The role of surface roughness in the measurement of slipperiness. Ergonomics. 44, 1200-1216 (2001). https://doi.org/10.1080/00140130110085565

44. Eaton, P., Paul, W.: Measuring AFM images. In: Atomic Force Microscopy. Oxford University Press, New York (2010)

45. Kim, I.J.: Investigation and Interpretation of Flooring Wear Development for Pedestrian Slip Resistance Assessments. Tribol. Trans. 61, 168-177 (2018). https://doi.org/10.1080/10402004.2017.1287318

46. ASTM F2508-12a: Standard Practice for Validation, Calibration, and Certification of Walkway Tribometers Using Referencre Surfaces. ASTM Int. (2012)

47. Aschan, C., Hirvonen, M., Mannelin, T., Rajama, E.: Development and validation of a novel portable slip simulator. Appl. Ergon. 36, 585-593 (2005). https://doi.org/10.1016/j.apergo.2005.01.015

48. Khanal, S.R., Gujrati, A., Vishnubhotla, S.B., Nowakowski, P., Bonifacio, C.S., Pastewka, L., Jacobs, T.D.B.: Characterization of small-scale surface topography using transmission electron microscopy. Surf. Topogr. Metrol. Prop. 6, 045004 (2018). https://doi.org/https:// doi.org/10.1088/2051-672X/aae5b3

49. Gujrati, A., Khanal, S.R., Pastewka, L., Jacobs, T.D.B.: Combining TEM, AFM, and Profilometry for Quantitative Topography Characterization Across All Scales. ACS Appl. Mater. Interfaces. 10, 29169 29178 (2018). https://doi.org/10.1021/acsami.8b09899

50. Prabhu, K.M.: Window Functions and Their Applications in Signal Processing. CRC Press, Boca Raton (2018)

51. Villarrubia, J.S.: Morphological estimation of tip geometry for scanned probe microscopy. Surf. Sci. 321, 287-300 (1994). https://doi.org/10.1016/0039-6028(94)90194-5

52. Church, E.L., Takacs, P.Z.: Effects of the nonvanishing tip size in mechanical profile measurements. Proc. SPIE 1332, Opt. Test. Metrol. III Recent Adv. Ind. Opt. Insp. 1332, 504-514 (1991)

53. Schmittbuhl, J., Vilotte, J.P.: Reliability of self-affine measurements. Phys. Rev. 51, 131-147 (1995)

54. Mandelbrot, B.B.: Self-affine fractals and fractal dimension. Phys. Scr. 32, 257-260 (1985). https://doi.org/10.1088/0031-8949/32/4/001

55. Gneiting, T., Schlather, M.: Stochastic models that separate fractal dimension and the Hurst effect. SIAM Rev. 46, 269-282 (2004). https://doi.org/10.1137/S0036144501394387

56. Falconer, K.J.: Fractal Geometry: Mathematical Foundations and Applications. Wiley, Hoboken (2015)

57. Brown, S.R., Scholz, C.H.: Broad bandwidth study of the topography of natural rock surfaces. J. Geophys. Res. 90, 12575-12582 (1985). https://doi.org/10.1029/jb090ib14p12575

58. Moghaddam, S.R.M., Acharya, A., Redfern, M.S., Beschorner, K.E.: Predictive multiscale computational model of shoe-floor coefficient of friction. J. Biomech. 66, 145-152 (2018).

https://doi.org/https://doi.org/10.1016/j.jbiomech.2017.11.009 